\title{
Genética dos transtornos afetivos
}

IVANor Velloso Meira Lima ${ }^{1}$

Everton Botelho Sougey ${ }^{1}$

Homero Pinto Vallada Filho ${ }^{2}$

\section{Resumo}

Os autores revisam neste artigo o conjunto de evidências genético-epidemiológicas que indicam a presença de fatores genéticos na vulnerabilidade para os transtornos afetivos. Apresentam também os dados obtidos até o momento, através de estratégias de genética molecular na busca de genes de susceptibilidade para o transtorno afetivo bipolar e para a depressão.

Palavras-chave: Transtorno afetivo bipolar, depressão, genética, estudos de ligação, estudos de associação.

\begin{abstract}
In this article the authors review the body of genetic-epidemiological evidences for the role of genes in mood disorders. Current molecular genetic studies searching for susceptibility genes for bipolar affective disorder and depression are also presented.
\end{abstract}

Key Words: Bipolar affective disorder, depression, genetics, linkage studies, association studies. 


\section{Introdução}

As doenças afetivas constituem síndromes caracterizadas por alterações patológicas do humor que pode variar desde uma extrema elação ou euforia até uma grave depressão ou disforia. Tais síndromes, particularmente aquelas caracterizadas por depressão, são bastante comuns e determinam importante prejuízo à sociedade, inclusive por associarem-se com elevadas taxas de suicídio.

A busca das bases etiológicas e fisiopatológicas dessas perturbações afetivas, visando à validação dos atuais constructos diagnósticos e à elaboração de estratégias terapêuticas e profiláticas mais efetivas, deve contemplar evidências clínicas disponíveis que apontam para o componente familiar como um dos principais fatores de risco para o aparecimento desses quadros (Goodwin e Jaminson, 1990).

Nesta revisão, pretendemos descrever sucintamente o corpo de evidências disponíveis que demonstram o envolvimento de fatores genéticos na vulnerabilidade para doenças afetivas, além de apresentar alguns dados obtidos até o momento através dos estudos moleculares, na busca de genes de suscetibilidade para o transtorno afetivo bipolar e para a depressão.

\section{Estudos genético-epidemiológicos}

Em regra, nos últimos 30 anos, os pesquisadores têm seguido a classificação sugerida por Leonhard (1957), que subdivide os pacientes com transtornos primários do humor em unipolares, no caso de só apresentarem depressão como alteração do humor, e bipolares, se acusam episódios de mania com ou sem depressão, ou ainda episódios de depressão com hipomania.

\section{Estudos com famílias}

Angst, em 1966 na Suíça, e Perris, no mesmo ano na Suécia, independentemente, apresentaram os primeiros resultados de estudos sistemáticos em famí-lias, utilizando a subdivisão de Leonhard. Ambos demonstraram agregação familiar das alterações do humor e, mais ainda, validaram a subdivisão da psicose maníaco-depressiva, encontrando maior freqüência de bipolares entre os familiares de bipolares e de unipolares entre os parentes de depressivos unipolares. A maioria dos estudos que se seguiram concordam com estes autores tanto na agregação familiar quanto na subdivisão (Revisado em Meira-Lima e Vallada, 1998).
Os resultados de estudos em famílias de pacientes com transtorno do humor podem ser sumarizados assim: o risco de parentes em primeiro grau de indivíduos não-afetados representativos da população geral é de quase $1 \%$ para doença bipolar e cerca de $5 \%$ para depressão unipolar. Quando comparamos este risco com o dos parentes em primeiro grau de pacientes com depressão, observamos que, para doença depressiva unipolar, o risco está aumentado aproximadamente três vezes, enquanto o risco de transtorno bipolar nesses familiares é quase o mesmo da população geral.

Finalmente, quando comparamos o grupo de familiares de indivíduos da população geral com o grupo de parentes em primeiro grau de portadores de transtorno bipolar, verificamos que o risco para a depressão unipolar está aumentado três vezes, enquanto o risco para doença bipolar nesses parentes está aumentado cerca de sete vezes. Com respeito à elevada prevalência de depressão entre familiares de bipolares, é importante lembrar que 10\% a 20\% dos pacientes bipolares apresentam os primeiros episódios como quadro depressivo (pseudo-unipolares) (Kendell, 1988).

\section{Estudos com gêmeos}

Entre os mais relevantes estudos com gêmeos nos transtornos do humor, podemos destacar:

- Bertelsen et al. (1977), investigaram na Dinamarca 123 pares de gêmeos e encontraram uma concordância, com relação à doença bipolar, de 79\% para gêmeos monozigóticos (MZ) e de 19\% para gêmeos dizigóticos (DZ), enquanto na depressão unipolar as taxas de concordância de MZ:DZ foram de 64\%:24\%.

- Torgersen (1986), em uma amostra norueguesa de 102 probandos predominantemente unipolares, encontrou um taxa de concordância em MZ:DZ de 51\%:20\%.

- McGuffin e Katz (1991) investigaram, na Inglaterra, 141 pares de gêmeos em sua maioria depressivos unipolares e observaram uma concordância entre MZ de $53 \%$ e em DZ de $28 \%$.

- Kendler et al. (1993), em uma amostra de 486 gêmeos suecos, verificaram uma concordância de $69 \%$ em pares MZ e de 34\% em pares DZ.

Podemos inferir, com base nos dados apresentados acima, que a taxa de concordância para problemas do humor em gêmeos MZ é duas a três vezes maior do que nos DZ, reforçando a hipótese de um componente genético nessas enfermidades.

A maioria dos estudos realizados com gêmeos, 
até o momento, permite estimar a herdabilidade na depressão unipolar em torno de $40 \%$, enquanto o transtorno afetivo bipolar teria uma herdabilidade de aproximadamente $70 \%$ a $80 \%$.

\section{Estudo com Adotados}

Até o presente, podemos identificar poucos relatos de investigações com adotados em distúrbios do humor, que são em sua maioria consistentes com a presença de um componente genético na determinação das enfermidades afetivas.

O primeiro estudo foi conduzido na Bélgica por Mendlewicz e Rainer (1977), que verificaram 29 adotados com doença maníaco-depressiva e observaram uma prevalência de transtornos afetivos em $31 \%$ dos pais biológicos desses indivíduos, comparada a uma prevalência de $12 \%$ nos pais adotivos.

Um segundo estudo nessa área foi conduzido por Cadoret (1978) que, estudando uma amostra de mães com doenças afetivas (bipolar e unipolar), evidenciou uma freqüência seis vezes maior de depressão em seus filhos adotados ao nascimento, comparados aos filhos de mães com outras condições clínicas também adotados ao nascimento.

Um outro estudo com adotados suecos, realizado por Von Knorring et al. (1983) encontrou poucas evidências de um componente genético em pais biológicos de 56 doentes afetivos adotados. Entretanto, questões metodológicas, como a baixa confiabilidade das informações de fichas de seguro-saúde que estes utilizaram em suas investigações, podem explicar a discrepância com os demais estudos. Finalmente, Wender et al. (1986) investigaram uma amostra dinamarquesa de 71 adotados afetados por transtornos de humor e relataram uma prevalência oito vezes maior de casos de depressão unipolar e quinze vezes maior de casos de suicídio nos pais biológicos desses indivíduos, comparados aos seus pais adotivos.

Apesar do número limitado de investigações com adotados, esses estudos são em sua maioria consistentes com a presença de um componente herdado na determinação dos transtornos afetivos.

\section{Estudos de Genética Molecular}

\section{Estudos de Ligação}

No que se refere ao uso de estudos de ligação nas doenças afetivas, o transtorno afetivo bipolar tem sido o mais freqüentemente estudado, provavelmente pela maior herdabilidade deste transtorno. A história dos estudos de ligação na doença bipolar, como bem definiu Risch e Botstein (1996) em sua revisão, assemelha-se muito ao curso da doença, com a euforia dos achados positivos, sendo logo seguida pela depressão da incapacidade de replicação dos mesmos.

A região com maior número de relatos de ligação com doença bipolar é a área subterminal do braço longo do cromossomo X (Xq28). Os primeiros estudos de Winokur e Tana (1969) e Mendlewicz e Fleiss (1974) demonstraram uma co-segregação da doença maníaco-depressiva com a cegueira para cores protan e deutan, um marcador clínico com localização cromossômica já definida naquela ocasião. Mendlewicz et al. (1980) pareceram confirmar este achado inicial, verificando ligação entre a deficiência da enzima glicose-6-fosfato desidrogenase (G-6-PD) com o distúrbio bipolar, entretanto, problemas metodológicos de caracterização dos afetados foram logo detectados nesse estudo. Baron (1987) obteve uma alta probabilidade de ligação ao X num estudo metodologicamente rigoroso conduzido em genealogias de judeus, porém ele mesmo reanalisou seus dados posteriormente com marcadores moleculares de DNA e não confirmou a ligação (Baron et al., 1993). Pekkarinen et al. (1995) relataram novas evidências de um gene para o transtorno bipolar no cromossomo X, na região q24-27. Entretanto, este resultado não foi confirmado em um outro estudo envolvendo 43 famílias inglesas e brasileiras (Vallada et al., 1997).

O primeiro relato de ligação positiva com marcadores moleculares do DNA ocorreu no estudo de Egeland et al. (1987) numa amostra da comunidade Amish da Pensilvânia. Nesse estudo, evidenciou-se ligação entre doença bipolar e marcadores da região $11 \mathrm{p} 15$. Outros pesquisadores não conseguiram replicar este achado, e o próprio grupo de Egeland, quando procurou reavaliar seus dados, verificou que o diagnóstico de alguns membros havia mudado, levando à diminuição da evidência inicial (Kelsoe et al., 1989).

Evidências sugestivas de ligação com a região pericentromérica do cromossomo 18 foram relatadas por Berretine et al. (1994 e 1997). Outro grupo independente encontrou também ligação com a região $18 p$ e também alguma evidência de ligação com a região 18q21; interessantemente esses achados ocorreram apenas nas famílias com transmissão paternal da doença (Stine et al.,1995). Esses relatos são difíceis de interpretar, sobretudo porque os marcadores utilizados cobriam regiões tão distantes uma das outras que os tornam inconsistentes na confirmação do achado inicial de ligação. 
Ainda sem nenhum achado definitivo, novos estudos com marcadores polimórficos mais adequados vêm sendo realizados, implicando as regiões cromossômicas 4p16, 12q23-q24, 21q22 e 22q12-q13 (Risch e Botstein, 1996; Kelsoe et al., 2001).

Em resumo, a proliferação de estudos, freqüentemente não replicados e mostrando aparente ligação, pode refletir a falta de homogeneidade na delimitação do fenótipo com interferências da comorbidade (coocorrência de mais de um diagnóstico no mesmo indivíduo) ou das fenocópias (manifestações semelhantes à doença em estudo, mas com origem nãogenética). Podem também ser resultado da própria complexidade etiológica da enfermidade que teria uma heterogeneidade genética ( $\mathrm{O}$ mesmo fenótipo resultaria de diferentes locus gênicos afetados em diferentes famílias) (Baron, 1997).

\section{Estudos de Associação}

Vários genes candidatos têm sido investigados para associação tanto no distúrbio bipolar quanto na depressão unipolar. Com base na hipótese de disfunção dos sistemas monoaminérgicos cerebrais na doença bipolar, o primeiro gene candidato investigado foi a tirosina hidroxilase, uma enzima que limita o ritmo de síntese das monoaminas. Leboyer et al. (1990) relataram associação de polimorfismos destes genes com doença maníaco-depressiva, contudo este achado não foi replicado por outros estudos.

Outras duas variantes genéticas relacionadas à hipótese monoaminérgica são:

$1^{\text {a }}$-Substituição do aminoácido valina por metionina na posição 108 (val108met) do gene, codificando a enzima catecol-O-metiltransferase (COMT) que afeta a atividade enzimática e que tem sido associada com pacientes bipolares cicladores rápidos e com depressão (Li et al., 1997; Kirov et al, 1998; Ohara et al, 1998), embora nenhuma associação com transtornos afetivos tenha sido detectada entre este polimorfismo e uma grande amostra de estudo multicêntrico (Biomed European Bipolar Collaborative Group, 1997).

$2^{\mathrm{a}}$ - Repetição polimórfica na região promotora do gene codificando a monoaminooxidase A (MAOA) que Lim et al. (1995) relataram estar associada ao transtorno bipolar, no que foram replicados por Preisig et al. (2000), embora relatos negativos também existam (Kunugi et al., 1999).

Em relação à depressão unipolar, apenas nos últimos anos têm surgido estudos moleculares, e estes têm focado principalmente os genes relacio- nados ao sistema serotoninérgico. Ogilvie et al. (1996) foram os primeiros a relatar uma associação entre um número variável de repetições em série (VNTR) no segundo intron do gene que codifica o transportador da serotonina (5-HTT) e depressão unipolar. Na tentativa de replicação desse estudo, Collier et al. (1996) não encontraram associação com depressão, mas verificaram uma freqüência maior de uma variante deste polimorfismo num grupo de doentes bipolares. Recentemente, uma variante que consiste na deleção de 44 pares de bases, na região do promotor do gene 5HTT que determina menor atividade transcricional no gene, foi associada, numa grande amostra populacional neozelandesa, com maior risco de depressão frente a eventos de vida (Caspi et al., 2003); esta mesma variante já havia sido relacionada com o transtorno bipolar em alguns estudos (Samochowiec et al., 2003), mas não em outros (Mendes de Oliveira et al., 1998).

Estudos de associação dos transtornos afetivos com variantes em outros genes não pertencentes aos sistemas de neurotransmissão monoaminérgicos também têm sido realizados. Entre alguns achados positivos não replicados podemos destacar associações com polimorfismos gênicos do sistema GABAergico (Oruc et al., 1997; Papadimitriou et al., 1998), com variantes em genes do sistema renina-angiotensina (Ariname et al., 1996; Meira-Lima et al., 2000), com loci contendo repetições trinucleotídicas (Lindblad et al., 1998; Meira-Lima, 2001) e com genes relacionados ao metabolismo de fosfolípides (Friedman et al., 2003; Meira-Lima et al., 2003).

Em síntese, os estudos de associação conduzidos até o presente não foram capazes de estabelecer resultados definitivos, haja vista que a maioria dos achados positivos não pôde ser replicada, o que sugere que possam tratar-se de falso-positivos decorrentes de estratificações populacionais ou do acaso (Carey, 1994).

O fato é que, em decorrência do vasto número de genes candidatos, a probabilidade, a priori, de que um dado gene investigado seja de fato relevante na determinação da doença é muito baixa (Crowe, 1993), o que nos indica um longo caminho pela frente na tentativa de uma investigação sistemática de todos os possíveis candidatos. 


\section{Conclusão}

Um consistente conjunto de evidências indica a existência de fatores genéticos na suscetibilidade para as doenças afetivas. Os dados disponíveis no momento não identificam de modo inequívoco nenhum gene de vulnerabilidade, mas a velocidade com que surgem novas tecnologias e abordagens na área de gené- tica molecular apontam que, provavelmente nos próximos anos, alguns dos genes relacionados à vulnerabilidade para a depressão e para o transtorno afetivo bipolar serão identificados. Muito provavelmente, essa identificação também propiciará uma revolução na prática clínica com os benefícios advindos da elucidação das vias bioquímicas relacionadas à patogênese dessas doenças afetivas.

\section{Referências Bibliográficas}

ANGST, J. - Zur Atiologie und Nozologie endogener depressiver Psychosen. In: Monographien aus der Neurologie und Psychiatrie. Springer-Verlag, Berlin, 112: 8, 1966.

Arinami, T.; Liming, L.; Mitsushio, H.; Itokowa, M.; Hamguchi, H.; Toru, M. An Insertion/Deletion Polymorphism in the Angiotensin Converting Enzyme Gene Is Associated with Both Brain Substance P Contents and Affective Disorders. Biol. Psychiatry 40: 1122-27, 1996.

Baron, M.; Risch, N.; Hamburger, R.; Mandel, B.; Kushner, S.; Newman, M.; Drumer, D.; Belmaker, R.H. - Genetic Linkage between X-Chromosome Markers and Bipolar Affective Illness. Nature 326:289-92, 1987.

Baron, M.; Freimer, N.F.; Risch, N.; Lerer, B.; Alexander, J.R.; Straub, R.E.; Asokan, S.; Das, K.; Peterson, A.; Amos, J. - Diminished support for linkage between manic depressive illness and X chromosome markers in three Israeli pedigrees. Nat. Genet .3: 49-55, 1993.

BARON, M. - Genetic linkage and bipolar affective disorder: progress and pitfall. Mol. Psychiatry. 2: 200-10, 1997.

Bertelsen, A.; Harvald, B.; Hauge, M.A. - A Danish Twin Study of ManicDepressive Disorders. Br. J. Psychiatry 130:330-51, 1977.

Berretine, W.H.; Ferraro, T.N.; Goldin, L.R.; Weeks, D.E.; Detera-Wadleigh, S.; Nurnberger, J.I.; Gershon, E.S. - Chromosome 18 DNA Markers and Manic-depressive Illness: Evidence for a Susceptibility Gene. Proc. Natl. Acad. Sci. 91: 5918-21, 1994.

Berretine, W.H.; Ferraro, T.N.; Goldin, L.R.; Detera-Wadleigh, S.; Choi, H.; Muniec, D.; Guroff, J..J.; Kazuba, D. M.; Nurnberger, J.I.; Hsieh, W.T.; Hoene, M.R.; Gershon, E.S.- A Linkage Study of Bipolar Illness. Arch. Gen. Psychiatry 54: 27-35, 1997.

CAdoret, R.J. - Evidence for Genetic Inheritance of Primary Affective Disorder in Adoptees. Am. J. Psychiatry 135:463-6, 1978.

Caspi, A.; Sugden, K.; Moffit, T.E.; Taylor, A.; Craig, I.W.; Harrigton, H.; Mcclay, J.; Mill, J.; Martin, J.; Braidwaite, A.; Poulton, R. - Influence of life stress on depression: moderation by a polymorphism in the 5HTT gene. Science 301(5631): 386-9, 2003.

Collier, D.A.; Stober, G.; Li, T.; Heils, A.; Catalano, M.; Di Bella, D.; ArranZ M.J.; Murray, R.M.; Vallada, H.P.; Bengel, D.; Muller, C.R.; Roberts, G.W.; Smeraldi, E.; Kirov, G.; Sham, P.; Lesch, K.P. - Susceptibility to Bipolar Affective Disorder and Unipolar Depression is Influenced by Allelic Variation of Functional Serotonin Transporter Expression. Mol. Psychiatry 1(6):453-60, 1996.

Crowe, R.R.- Candidate Genes in Psychiatry: An Epidemiological Perspective. Am. J. Med. Genet. 48:74-7, 1993.

Egeland, J.A.; Gerhard, D.S.; Pauls, D.L.; Sussex, J.N.; Kidd, K.K.; Allen, C.R.; Hostetter, A.M.; Housman, D.E. - Bipolar Affective Disorders Linked to DNA Markers on Chromosome 11. Nature 325: 783-7, 1987.

Fridman, C.; OJopi, E.P.; Gregorio, S.P.; Ikenaga, E.H.; Moreno, D.H.; Demetrio, F.N.; Guimaraes, P.E.; Vallada, H.P.; Gattaz, W.F.; Dias Neto, E. Association of a new polymorphism in ALOX12 gene with bipolar disorder. Eur Arch Psychiatry Clin Neurosci. 253(1): 40-3, 2003.

Goodwin, F.K.; Jamison, K.R. - Manic-Depressive Illness. Oxford University Press, New York, 1990.

Kelsoe, J.R.; Ginns, E.I.; Egeland, J.A.; Derhard, D.S.; Goldstein, A.M.; Bale,
S.J.; Pauls, D.L.; Long, R.T.; Kidd, K.K.; Conte, G.; Housman, D.E.; Paul, S.M. - Re-evaluation of the Linkage Relationship between Chromosome 11p Loci and the Gene for Bipolar Affective Disorder in Old Order Amish. Nature 342:238-43, 1989.

Kelsoe, J.R.; Spence, M.A.; Loetscher, E.; Foguet, M.; Sadovnick, A.D.; Remick, R.A.; Flodman, P.; Khristich, J.; Mroczkowski-Parker, Z.; Brown, J.L.; MASSER, D.; Ungerleider, S.; RAPAPORT, M.H.; Wishart, W.L.; LuebBert, H. A genome survey indicates a possible susceptibility locus for bipolar disorder on chromosome 22. Proc. Natl.Acad. Sci. USA 98(2): 585-90, 2001

Kendell, R.E.; Zealley, A.K. - Companion to Psychiatric Studies, 4th ed. Chuchill Livingstone, Edinburgh, UK, 1988.

Kendler,K.S.; Pederson, N.; Johnson, L.; Neale, M.C.; Mathe, A.A.- A Pilo Swedish Twin Study of Affective Illness, Including Hospital-andpopulation- Ascertained subsamples. Arch. Gen. Psychiatry 50:699-706, 1993.

Kunugi, H.; Vallada, H.P.; Hoda, F.; Kirov, G.; Gill, M.; Aitchison, K.J.; Ball D.; Arranz, M.J.; Murray, R.M.; Collier, D.A. - No evidence for an association of affective disorders with high or low-activity allele of catechol-O-methyltransferase gene. Biol Psychiatry 42: 282-5, 1997.

Kunugi, H.; Ishida, S.; Kato, T.; Tatsumi, M.; SaKai, T.; Hattori, M.; Hirose, T. NANKO, S.A. - Functional Polymorphism in the Promoter Region of Monoamine Oxidase-A Gene and Mood Disorders. Mol Psychiatry 4(4):393-5, 1999.

Leboyer, M.; Malafosse, A.; Boularand, S.; Campion, D.; Gheysen, F.; Samolyk, D.; Henriksson, B.; Denise, E.; Des Lauries, A.; Lepine, J.P.; Zarifian, E. Clerget-Darpoux, F.; Mallet, J. - Tyrosine Hydroxylase Polymorfisms Associated With Manic-Depressive Illness. Lancet 335; 1219, 1990.

Leonhard, K. - The Classification of Endogenous Psychoses. Robins E. ed. New York, Irvington, 1957.

Li, T.; Vallada, H.; Curtis, D.; Arranz, M.; Xu, K.; Cai, G.; Deng, M.; Liu, J. Murray, R.; Liu, X.; Collier, D.A. - Catechol-O-methyltransferase Val158Met polymorphism: frequency analysis in Han Chinese subjects and allelic association of low activity allele with bipolar affective disorder. Pharmacogenetics 7(5): 344-53, 1997.

Lindblad, K.; Nylander, P.O.; Zander, C.; Yuan, O.P.; Stahle, L.; Engstrom, C.; Balciuniene, J.; Pettersson, U.; Breschel, T.; Mcinnis, M.; Ross, C.A Adolfsson, R.; Schalling, M. - Two commonly expanded CAG/CTG repeat loci: involvement in affective disorders? Mol Psychiatry 3: 405-10, 1998.

Lim, L.C.; Powell, J.F.; Sham, P.; Castle, D.; Hunt, N.; Murray, R.M.; Gill, M. - Evidence for a Genetic Association between Alleles of Monoamine Oxidase A Gene and Bipolar Affective Disorder. Am. J. Hum. Genet. 54:1122-4, 1995

McGuffin, P.; Katz, R. - Nature, Nurture and Depression: A Twin Study. Psychological Med. 21; 239-45, 1991.

Meira-Lima, I.V.; Vallada, H. - Estudos Genéticos no Transtorno Afetivo Bipolar. Revista de Psiquiatria Clínica 25 (4):166-75, 1998.

Meira-Lima, I.V.; Pereira, A.C.; Motta, G.F.; Krieger, J.E.; Vallada, H. Angiotensinogen and Angiotensin Enzyme Converting Gene Polymorphism and the Risk of Bipolar Affective Disorder. Neuroscience Letters 293 (2): 103-6, 2000. 
Meira-Lima, I.V.; Zhao, J.; Sham, P.; Pereira, A.C.; Krieger, J.E.; Vallada, H. - Association and Linkage Studies Between Bipolar Affective Disorder and the Polymorphics CAG Repeat Loci ERDA1, SEF21b, MAB21L and KCNN3. Molecular Psychiatry 6: 565-9, 2001.

Meira-Lima, I.V.; Jardim, D.; Jungueira, R.; Ikenaga, E.; Vallada, H. - Allelic association study between phospholipase A2 genes and bipolar affective disorder. Bipolar Disord. 5(4):295-9, 2003

Mendes de Oliveira, Jr.; Otto, P.A.; Vallada, H.; Lauriano, V.; Elkis, H.; Lafer, B.; Vasquez, L.; Gentil, V.; Passos-Bueno, M.R.; Zatz, M. Analysis of a novel functional polymorphism within the promoter region of the serotonin transporter gene (5-HTT) in Brazilian patients affected by bipolar disorder and schizophrenia. Am. J. Med. Genet. 81(3):225-7, 1998.

Mendlewicz, J.; Fleiss, J.L. - Linkage studies With Chromosome Markers in Bipolar (Manic-Depressive) and Unipolar (Depressive) Illness. Biol. Psychiatry 9: 261-94;,1974.

Mendlewicz, J.; Rainer, J.D. - Adoption Study Supporting Genetic Transmission in Manic-Depressive Illness. Nature 268: 327-9, 1977.

Mendlewicz, J.; Linkowsk, P.; Wilmotte, J. - Linkage between Glucose-6Phosphate Dehydrogenase Deficiency and Manic-Depressive Psychosis. Br. J. Psychiatry 137: 337-42, 1980.

Ogilvie, A.D.; Battersby, S.; Bubb, V.J.; Fink, G.; Harmar, A.J.; Goodwin, G.M.; SMiтh, C.A.D. - Polymorphism in Serotonin Transporter Gene Associated with Susceptibility to Major Depression. Lancet 347: 7313, 1996 .

Ohara, K.; Suzuki, Y.; Yoshida, K.; Ohara, K. - Anticipation and Imprinting in Japanese Familial Mood Disorders. Psychiatry Res. 79(3):191-8, 1998.

Oruc, L.; Verheyen, G.R.; Furac, I.; Ivezic, S.; Jakovljevic, M.; Raeymaekers, P.; Van Broeckhoven, C. - Positive association between the GABRA5 gene and unipolar recurrent major depression. Neuropsychobiology 36(2):62-4, 1997.

Papadimitriou, G.N.; Dikeos, D.G.; Karadima, G.; Avramopoulos, D.; Daskalopoulou, E.G.; Vassilopoulos, D.; Stefanis, C.N. - Association between the $\mathrm{GABA}(\mathrm{A})$ receptor alpha5 subunit gene locus (GABRA5) and bipolar affective disorder. Am J Med Genet 81(1): 73-80, 1998 .

Pekkarinen, P.; Terwilliger, J.; Bredbacka, P.; Hovatta, L.; Lonnqvuist, J.; Peltonen, L. - Evidence of a predisposing locus to bipolar disorder on Xq24-q27.1 in a extended Finnish pedigree. Genome Research 5: $105-15,1995$.
Perris, C. - A Study of Bipolar (Manic-Depressive) and Unipolar Recurrent Depressive Psychoses. Acta Psychiatrica Scand. 42:1-189, 1966.

Preisig, M.; Bellivier, F.; Fenton, B.T.; Baud, P.; Berney, A.; Courtet, P.; Hardy, P.; Golaz, J.; Leboyer, M.; Mallet, J.; Matthey, M.L.; Mouthon, D.; Neidhart, E.; Nosten-Bertrand, M.; Stadelmann-Dubuis, E.; Guimon, J.; Ferrero, F.; Buresi, C.; Malafosse, A. - Association Between Bipolar Disorder and Monoamine Oxidase A Gene Polymorphisms: Results of a Multicenter Study. Am J Psychiatry 157(6): 948-55, 2000.

Risch, N.; Boltstein, D. - A Manic Depressive History. Nat. Genet. 12: 351-3. 1996.

Samochowiec, J.; Czerski, P.M.; Ostapowicz, A.; Chlopocka, M.; Mcclay, J.; Horodnick, J.; RYвакоWSкI, J.K. - Association analysis of the insertion/ deletion polymorphism in serotonin transporter gene in patients with affective disorder. Eur. Psychiatry 18(3): 129-32, 2003

Stine, O.C.; Xu, J.; Koskela, R.; Mcmahon, F.J.; Gschwend, M.; Friddle, C.; Clark, C.D.; Mcinnis, M,; Simpson, S.; Breschel, T.S.; Vishio, E.; Riskin, K.; Feilotter, H.; Chen, E.; Shen, S.; Folstein, S.; Meyers, D.A.; Botstein, D.; Marr, T.G.; Depaulo, Jr. - Evidence for Linkage of Bipolar Disorder to Chromosome 18 with Parent-of-Origin Effect. Am. J. Hum. Genet. 57: 1384-94.

Torgersen, S. (1986) - Genetic Factors in Moderately Severe and Mild Affective Disorders. Arch. Gen. Psychiatry 43:222-6, 1995.

Vallada, H.; Craddock, N.; Vasoues, L.; Curtis, D.; Kirov, G.; Lauriano, V.; Gentil, V.; Passos-Bueno, R.; Murray, R.M.; Zatz, M.; Mcguffin, P.; Powell, J.F.; Gill, M.; Owen, M.; Collier, D.A. - Linkage Studies in Bipolar Affective Disorder With Markers on Chromosome 21. J. Affec. Dis. 41: 217-21, 1996.

Vallada, H.; Vasques, L.; Curtis, D.; Zatz, M.; Kirov, G.; Lauriano, V.; Gentil, V.; Murray, R.M.; Mcguffin, P.; Owen, M.; Gill, M.; Craddock, N.; Collier, D.A. - Linkage Studies in Bipolar Affective Disorder With Markers on Chromosome Xq25-27. Psychiat. Genet. 8(3): 183-6, 1999.

Von Knorring, A.L.; Cloninger, C.R.; Bohman, M.; Sigvardsson, S. - An Adoption Study of Depressive Disorders and Substance Abuse. Arch. Gen. Psychiatry 40: 943-50, 1983.

Wender, P.H.; Kety, S.S.; Rosenthal, D.; Schulsinger, F.; Ortmann, J.; Lunde, I. - Psychiatrics Disorders in the Biological and Adoptive Families of Adopted Individuals with Affective Disorders. Arch. Gen. Psychiatry 43: 923-9, 1986.

Winokur, G.; TANA, V.L. - Possible Role of X-Linked Dominant Factor in Manic-Depressive Disease. Dis. Nerv. System 30: 89-93, 1969. 